\title{
Investigation on Beamspace Multiple-Input Multiple-Output Synthetic Aperture Radar Data Imaging
}

\author{
Hongbo Mo, ${ }^{1}$ Wei Xu, ${ }^{2}$ and Zhimin Zeng ${ }^{1}$ \\ ${ }^{1}$ School of Information and Communication Engineering and Beijing Key Laboratory of Network System Architecture and Convergence, \\ Beijing University of Posts and Telecommunications, Beijing 100876, China \\ ${ }^{2}$ Department of Spaceborne Microwave Remote Sensing, Institute of Electronics, Chinese Academy of Sciences (IECAS), \\ Beijing 100190, China
}

Correspondence should be addressed to Hongbo Mo; mohongbo@bupt.edu.cn

Received 3 October 2015; Revised 4 January 2016; Accepted 27 January 2016

Academic Editor: Angelo Liseno

Copyright (C) 2016 Hongbo Mo et al. This is an open access article distributed under the Creative Commons Attribution License, which permits unrestricted use, distribution, and reproduction in any medium, provided the original work is properly cited.

\begin{abstract}
The multiple-input multiple-output (MIMO) technique can improve the high-resolution wide-swath imaging capacity of synthetic aperture radar (SAR) systems. Beamspace MIMO-SAR utilizes multiple subpulses transmitted with different time delays by different transmit beams to obtain more spatial diversities based on the relationship between the time delay and the elevation angle in the side-looking radar imaging geometry. This paper presents a beamspace MIMO-SAR imaging approach, which takes advantage of real time digital beamforming (DBF) with null steering in elevation and azimuth multichannel raw data reconstruction. Echoes corresponding to different subpulses in the same subswath are separated by DBF with null steering onboard, while echoes received and stored by different azimuth channels are reconstructed by multiple Doppler reconstruction filters on the ground. Afterwards, the resulting MIMO-SAR raw data could be equivalent to the raw data of the single-channel burst mode, and classical burst mode imaging algorithms could be adopted to obtain final focused SAR images. Simulation results validate the proposed imaging approach.
\end{abstract}

\section{Introduction}

High-resolution wide-swath (HRWS) imaging capacity is one of the most important aims of future spaceborne microwave remote sensing $[1,2]$. To overcome the inherent limitation between azimuth resolution and unambiguous swath width in conventional single-channel spaceborne synthetic aperture radar (SAR) systems [2], multiple innovative multichannel imaging modes are proposed and investigated [3-17]. The concept of multiple-input multiple-output (MIMO) SAR for HRWS imaging is hotly discussed and investigated in recent years [8-17]. Compared with single-input multipleoutput (SIMO) SAR systems, multiple waveforms are instantaneously transmitted by multiple transmitters to illuminate the same imaged swath and their corresponding echoes are received by multiple subapertures. As a result, a larger number of obtained azimuth samples in a single pulse repetition interval could be used to enhance performances of spaceborne SAR systems including geometric resolution, swath width, ambiguity to signal ratio (ASR), and signal to noise ratio (SNR).

According to different types of transmitted waveforms, MIMO-SAR could be characterized as orthogonal waveform MIMO-SAR, subband MIMO-SAR, and beamspace MIMOSAR [15]. Different from other types of MIMO-SAR systems, multiple waveforms with the same phase coding and carrier frequency are transmitted in beamspace MIMO-SAR. Different transmitted subpulses are transmitted with different elevation beams and time delays, and then their corresponding echoes could be separated by digital beamforming (DBF) in elevation based on the relation between the short transmitted time delay and the elevation angle in the side-looking SAR imaging geometry [15]. As a result, more spatial diversities would be obtained to improve the high-resolution wideswath imaging capacity.

In this paper, a processing approach for beamspace MIMO-SAR data focusing is proposed. The proposed approach includes three important steps: DBF with null 
steering onboard, multichannel azimuth data reconstruction, and single-channel burst mode SAR imaging. The key point of the proposed approach is DBF onboard and azimuth multichannel reconstruction for multichannel raw data preprocessing. The DBF step with null steering operated onboard separates echoes corresponding to different subpulses to extract more spatial diversities. Compared with the conventional DBF on receive approach named as scan-onreceive (SCORE) [6,7], the modified DBF approach with null steering is implemented by two complex matrix multiplications. Afterwards, echoes with different spatial diversities stored in all azimuth channels are reconstructed to overcome the nonuniform azimuth sampling $[18,19]$. Compared with the azimuth multichannel reconstruction algorithm in $[18$, 19], the reconstruction matrix in the proposed approach is also modified according to the azimuth imaging geometry of beamspace MIMO-SAR. Finally, the resulting raw data after DBF onboard and azimuth multichannel reconstruction could be focused by classical burst mode imaging algorithms [20-25].

This paper is organized as follows. Section 2 reviews the imaging scheme of beamspace MIMO-SAR and real time DBF on receiving chirp signals. Section 3 is focused on presenting the proposed imaging approach to handle the beamspace MIMO-SAR raw data. Simulation results on point targets are given in Section 4 to validate the proposed approach. Finally, this paper is concluded in Section 5.

\section{Beamspace MIMO-SAR and DBF in Elevation}

2.1. Beamspace MIMO-SAR Imaging Scheme. For future spaceborne microwave remote sensing missions, SAR sensors will require a complete and frequent coverage of the Earth with a reasonably high geometric resolution, for example, an imaged swath width of no less than $400 \mathrm{~km}$ with an azimuth resolution of below $5 \mathrm{~m}$ [25]. To implement such great imaging capacity, beamspace MIMO-SAR takes advantage of intrapulse beamsteering on transmit, the displaced phase center multiple azimuth beam (DPCMAB) technique, and DBF on receive and burst imaging scheme. Figure 1 demonstrates a beamspace MIMO-SAR system example with two transmit subapertures (Tx) and four receive subapertures $(\mathrm{Rx})$. For the first subswath, two transmit subapertures in azimuth transmit radar subpulses with different time delays in sequences and echoes corresponding to different subpulses are received by different receive subapertures in azimuth. As a result, for the first subswath as shown in Figure 1, spatial diversity a (SD-a) corresponding to the first transmitted subpulse a and SD-d corresponding to the second transmitted subpulse $d$ are obtained by all receive subapertures in azimuth. For the third subswath illuminated by another two subpulses $b$ and $c$ via the second elevation transmitting beam as shown in Figure 1, both diversities of SD-b corresponding to the transmitted subpulse $\mathrm{b}$ and SDc corresponding to the transmitted subpulse $c$ are obtained by all receive subapertures in azimuth. Consequently, such beamspace MIMO-SAR system with two Tx and four $\mathrm{Rx}$ could obtain eight individual spatial samples in a single pulse repetition interval (PRI), and two of them are superposed as shown in Figure 1. As two subpulses are transmitted by each Tx to illuminate two subswaths, the four-subswath imaging scheme is just implemented by the two-burst mode as shown in Figure 1. Consequently, compared with other imaging modes with the same antenna, the high-resolution wide-swath imaging capacity is obviously improved due to the increased spatial samples in azimuth in a single PRI and the reduced number of bursts for the same swath width.

As shown in Figure 1(a), four subpulses are transmitted in a single PRI and their corresponding echoes will overlap in the receiving window. However, four subpulses are transmitted with different time delays or/and with different elevation transmitting beams and this scheme leads to the fact that the raw data recorded in the same position of the receiving window are echoes corresponding to different subpulses simultaneously arriving at the receiving antenna from multiple directions. Echoes from different directions could be separated by DBF in elevation. The DBF operation leads to the fact that echoes from the desired arriving direction are received with a high antenna gain, while other simultaneously arriving echoes are received with a very low antenna gain and could even be neglected. To obtain more spatial diversities, multiple beams with different pointing directions in elevation are adopted to transmit different subpulses and receive their corresponding echoes, and this is why this imaging scheme is named as beamspace MIMO-SAR.

Echoes from different subswaths could be easily separated by DBF in elevation, since the difference between their arriving directions is much larger than the beamwidth of the narrow scanning receive beam. Unfortunately, echoes of different subpulses transmitted into the same subswath are difficult to be separated by the conventional DBF receive approach, since the small angular interval between their echoes arriving directions is caused by the short time delay between two transmitted subpulses such as point targets $\mathrm{P}$ and $\mathrm{Q}$ as shown in Figure 2. To resolve the range ambiguities from different subpulses in the same subswath, the height $h_{\text {ant }}$ of the large receive antenna should be [9]

$$
h_{\mathrm{ant}} \geq \frac{2 \lambda \cdot r_{\mathrm{far}} \cdot \tan \left(\theta_{\mathrm{inc}, \max }\right)}{c \cdot \tau_{d}},
$$

where $\tau_{d}$ is the time delay of two transmitted subpulses as shown in Figure 1(a), $\lambda$ is the wavelength, $r_{\text {far }}$ indicates the far range, $c$ is the light speed, and $\theta_{\text {inc,max }}$ denotes the largest incident angle. Equation (1) demonstrates that the very large receive antenna is required for echo receiving, especially for the farther subswath, and the height $h_{\text {ant }}$ of the large receive antenna is only a little larger than the minimum requirement of (1). To improve the quality of echoes separating corresponding to different subpulses from the same subswath, DBF with null steering instead of conventional DBF could be adopted.

To implement the beamspace MIMO-SAR imaging scheme, two subpulses should be transmitted into different subswaths by the same Tx, and echoes of multiple subpulses are, respectively, received by different narrow scanning receive beams. Furthermore, echoes are individually received 


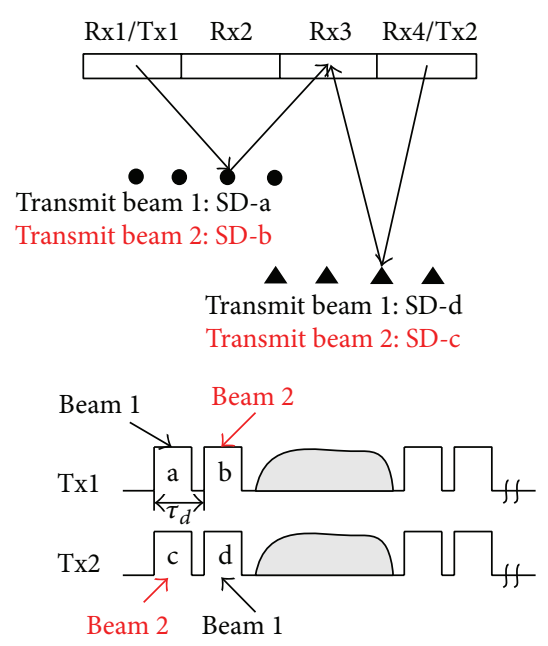

(a) In azimuth

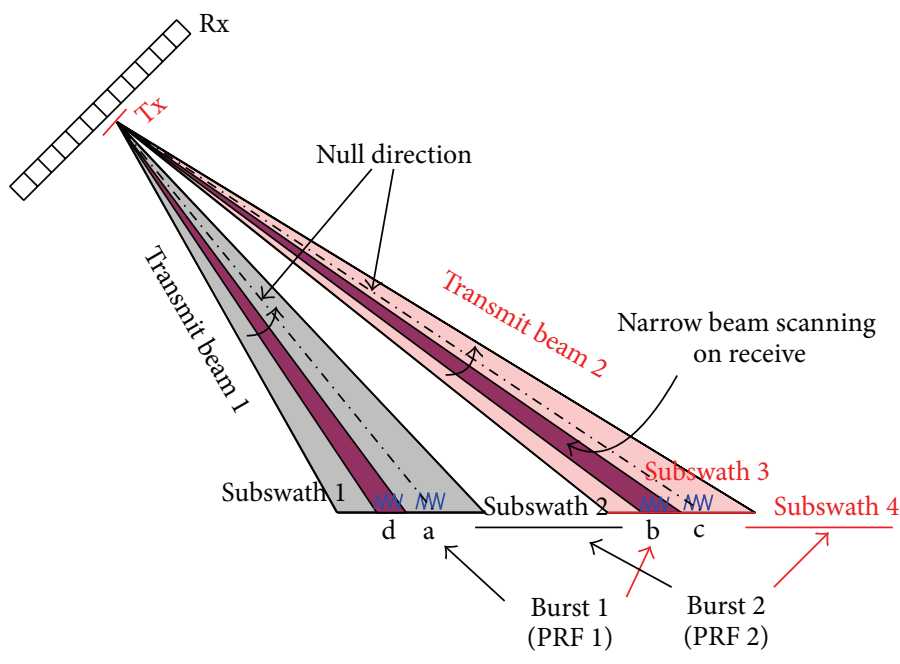

(b) In elevation

FIGURE 1: Beamspace MIMO-SAR imaging scheme. (a) Four subpulses are transmitted by two subapertures in a single PRI, which results in more spatial diversities (SDs) in azimuth. (b) Two subpulses transmitted by the same Tx are used to illuminate different subswaths, while echoes corresponding to subpulses with different time delays transmitted by different subapertures are separated by narrow beam scanning on receive with null steering.

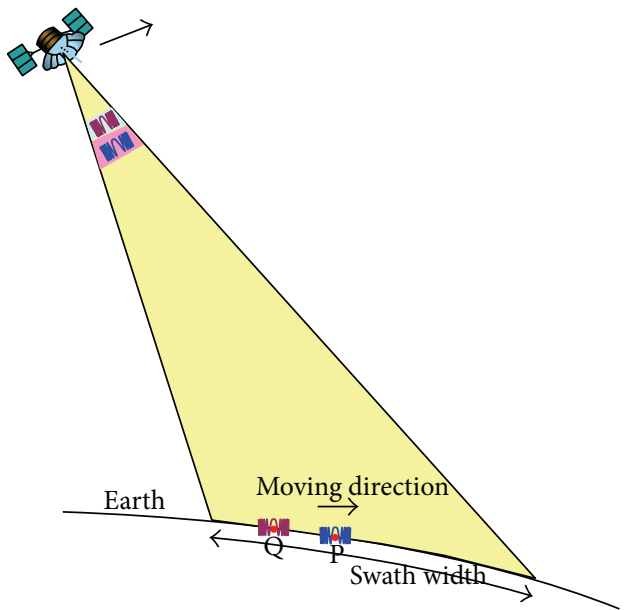

(a)

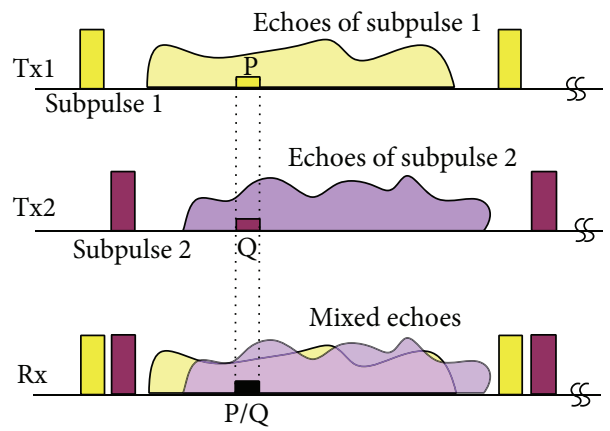

(b)

FIGURE 2: Two subpulses transmitted in a single PRI to illuminate the same swath and their corresponding echoes superimpose in the receiving window. (a) Imaging geometry of two subpulses in a single PRI to illuminate the same swath. (b) Echoes of different subpulses superimpose in the receiving window.

by multiple subapertures arranged in azimuth. Therefore, a large receive antenna, which is divided into multiple subapertures in both azimuth and elevation, is required in a beamspace MIMO-SAR system. Two subapertures are used to transmit radar pulses and receive backscatter echoes, while others are only used to receive radar echoes. Furthermore, each subaperture contains lots of element antennas as shown in Figure 3(a). Intrapulse beamsteering in elevation to transmit different subpulses into different subswaths in a single PRI is implemented by analog beamforming (ABF) controlled by a set of phase shifters as shown in Figure 3(b). Sharp high gain scanning pencil beam with null steering is implemented by DBF processing of multiple channel raw data in elevation as shown in Figure 3(c).

2.2. DBF on Receive in Elevation. In beamspace MIMOSAR systems, subpulses with different time delays and different beam pointing directions are transmitted, and their corresponding echoes could be separated by DBF on receive in elevation. The conventional DBF on receive onboard 


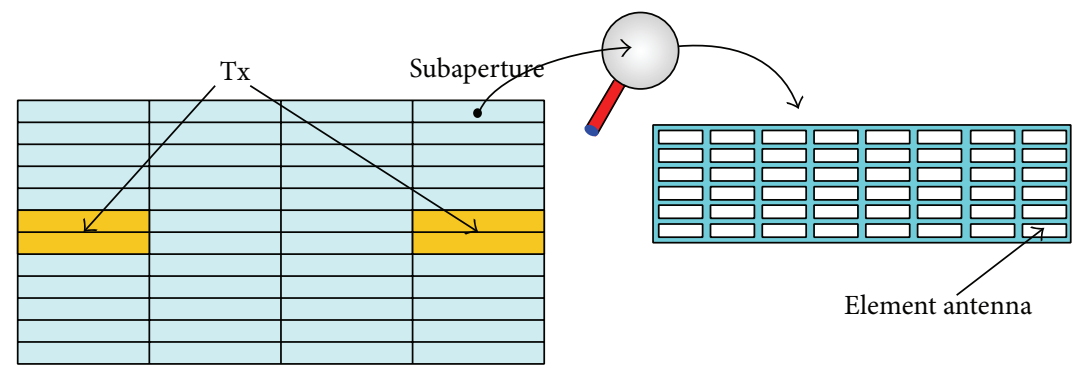

The whole receive antenna $(\mathrm{Rx})$

(a)

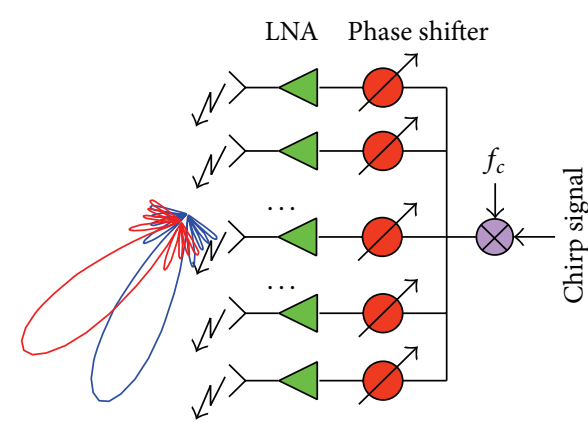

(b)

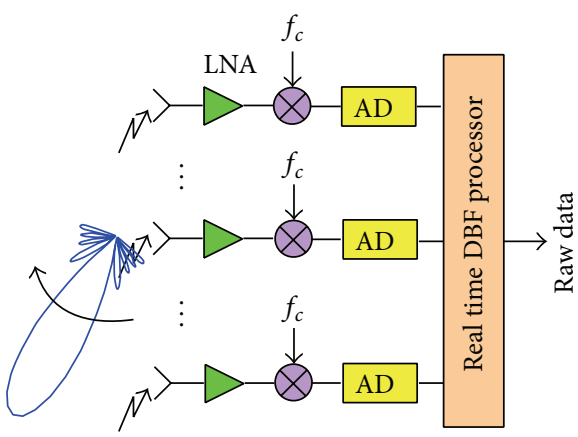

(c)

FIGURE 3: A large antenna adopted in beamspace MIMO-SAR. (a) The large receive antenna is divided into multiple subapertures in both azimuth and elevation directions, and each subaperture contains lots of element antenna. (b) Intrapulse beamsteering to transmit multiple subpulses via the ABF net. (c) Sharp pencil beam to receive echoes by a real DBF processor.

technique is detailed and investigated in $[6,7,26]$. In this novel receive technique, signals received by multiple subapertures in elevation are combined into one to form a sharp and high gain receive beam in real time. The following chirp signal is assumed in the proposed SAR system:

$$
s(\tau)=\operatorname{rect}\left[\frac{\tau}{\tau_{p}}\right] \cdot \exp \left(j 2 \pi f_{c} \tau+j \pi k_{r} \tau^{2}\right),
$$

where $\tau$ indicates the fast time in elevation, $\tau_{p}$ is the pulse duration, $f_{c}$ is the carrier frequency, and $k_{r}$ is the linear frequency modulation rate of the transmitted pulse. The received signal in the $k$ th subaperture from a point target is as follows:

$$
\begin{aligned}
s_{k}(\tau)= & \operatorname{rect}\left[\frac{\tau-\tau_{k}}{\tau_{p}}\right] \\
& \cdot \exp \left[j 2 \pi f_{c}\left(\tau-\tau_{k}\right)+j \pi k_{r}\left(\tau-\tau_{k}\right)^{2}\right]
\end{aligned}
$$

with

$$
\tau_{k}=\tau_{0}-\Delta \tau_{k}=\tau_{0}-\frac{(k-1)}{c} \cdot d \cdot \sin \left(\theta_{0}\right),
$$

where $d$ is the distance between the phase centers of two adjacent subapertures in elevation, $\theta_{0}$ indicates the actual direction of arrival (DOA) of the received echoes, and $\tau_{0}$ denotes the time delay of the first subaperture in elevation. According to the working principle of the phased array antenna, the phase weighting factor corresponding to the $k$ th subaperture in elevation to form the sharp scanning beam is as follows:

$$
w_{k}(\tau)=\exp \left\{-j 2 \pi \frac{(k-1) \cdot d}{\lambda} \cdot \sin [\theta(\tau)]\right\},
$$

where $\theta(\tau)$ is the steering angle as a function of the fast time $\tau[6,7]$. Afterwards, the final obtained demodulated baseband signal of the $k$ th subaperture in elevation can be expressed as follows:

$$
\begin{aligned}
& s_{k}(\tau) \\
& =\exp \left(-j 2 \pi f_{c} \tau_{0}\right) \cdot \operatorname{rect}\left[\frac{\tau-\left(\tau_{0}-\Delta \tau_{k}\right)}{\tau_{p}}\right] \\
& \quad \cdot \exp \left\{j \pi k_{r}\left[\tau-\left(\tau_{0}-\Delta \tau_{k}\right)\right]^{2}\right\} \\
& \quad \cdot \exp \left\{-j 2 \pi \cdot \frac{(k-1) \cdot d}{\lambda} \cdot\left[\sin (\theta(\tau))-\sin \left(\theta_{0}\right)\right]\right\} .
\end{aligned}
$$

Due to the very limited angular interval for a subswath in spaceborne SAR, the sine of the scanning angle $\theta(\tau)$ in (6) can be expressed as a linear approximation of the fast time $\tau$ and 
evaluated at the time delay $\tau_{c}$ [7]. Consequently, the received signal of the $k$ th subaperture in elevation in (6) is rewritten as

$$
\begin{aligned}
& s_{k}(\tau) \approx \exp \left(-j 2 \pi f_{c} \tau_{0}\right) \cdot \operatorname{rect}\left[\frac{\tau-\left(\tau_{0}-\Delta \tau_{k}\right)}{\tau_{p}}\right] \\
& \cdot \exp \left\{j \pi k_{r}\left[\tau-\left(\tau_{0}-\Delta \tau_{k}\right)\right]^{2}\right\} \\
& \cdot \exp \left\{-j 2 \pi \frac{(k-1) \cdot d}{\lambda} \cdot \cos \theta\left(\tau_{c}\right)\right. \\
& \left.\cdot \frac{\partial \theta\left(\tau_{c}\right)}{\partial \tau}\left(\tau-\tau_{0}\right)\right\}=\exp \left(-j 2 \pi f_{c} t_{0}\right) \\
& \operatorname{rect}\left[\frac{\tau-\left(\tau_{0}-\Delta \tau_{k}\right)}{\tau_{p}}\right] \\
& \cdot \exp \left\{j \pi k_{r}\left[\tau-\left(\tau_{0}-\Delta \tau_{k}\right)\right]^{2}\right\} \\
& \cdot \exp \left\{-j 2 \pi \frac{(k-1) \cdot d}{\lambda} \cdot \cos \theta\left(\tau_{c}\right) \cdot \frac{\partial \theta\left(\tau_{c}\right)}{\partial \tau}\right. \\
& \left.\cdot\left[\tau-\left(\tau_{0}-\Delta \tau_{k}\right)\right]\right\} \cdot \exp \left\{j 2 \pi \cdot \frac{(k-1) \cdot d}{\lambda}\right. \\
& \left.\cdot \cos \theta\left(\tau_{c}\right) \cdot \frac{\partial \theta\left(\tau_{c}\right)}{\partial \tau} \cdot \Delta \tau_{k}\right\}
\end{aligned}
$$

where $\tau_{c}$ is the time delay for the middle slant range in a subswath. Using the principle of stationary phase (POSP), the spectrum of the received signal in (7) could be easily obtained and expressed as follows:

$$
\begin{aligned}
& S_{k}(f) \\
& =\exp \left(-j 2 \pi f_{c} \tau_{0}\right) \cdot \operatorname{rect}\left[\frac{f+(k-1) \cdot f_{0}}{k_{r} \tau_{p}}\right] \\
& \cdot \exp \left\{-j \frac{\pi}{k_{r}}\left[f+(k-1) \cdot f_{0}\right]^{2}\right\} \\
& \cdot \exp \left\{-j 2 \pi \cdot f \cdot\left(\tau_{0}-\Delta \tau_{k}\right)\right\} \\
& \quad \cdot \exp \left\{j 2 \pi \cdot \frac{(k-1) \cdot d}{\lambda} \cdot \cos \theta\left(\tau_{c}\right) \cdot \frac{\partial \theta\left(\tau_{c}\right)}{\partial \tau} \cdot \Delta \tau_{k}\right\} \\
& =C_{k} \cdot \exp \left\{-j 2 \pi\left(f+f_{c}\right) \tau_{0}\right\} \\
& \quad \cdot \operatorname{rect}\left[\frac{f+(k-1) \cdot f_{0}}{k_{r} \tau_{p}}\right] \cdot \exp \left\{-j \frac{\pi}{k_{r}} f^{2}\right\} \\
& \quad \cdot \exp \left\{j 2 \pi f\left(\Delta \tau_{k}-\frac{(k-1)}{k_{r}} \cdot f_{0}\right)\right\}
\end{aligned}
$$

with

$$
f_{0}=\frac{d}{\lambda} \cdot \cos \theta\left(\tau_{c}\right) \cdot \frac{\partial \theta\left(\tau_{c}\right)}{\partial \tau}
$$

where $C_{k}$ is a complex constant. In order to compensate the spread of the signal over time of the chirp signal, from (8), it can be seen that a linear phase term should be compensated via the following function:

$$
H_{k}(f)=\exp \left\{-j 2 \pi f\left(\Delta \tau_{k}-\frac{(k-1) f_{0}}{k_{r}}\right)\right\} .
$$

This linear phase term compensation could also be implemented in the time domain via the time delay $D_{k}$, and it can be expressed as follows:

$$
D_{k}=\Delta \tau_{k}-\frac{(k-1) f_{0}}{k_{r}} .
$$

After the modification of the spectrum realized by the short time delay $D_{k}$ for each subaperture in elevation, all signals will be combined together. Therefore, a sharp high gain scanning beam to receive echoes is implemented via a multiplied vector and a set of time delayers. The block diagram of the real time conventional DBF processor in elevation onboard is shown in Figure 4.

\section{Imaging Approach}

The above-mentioned beamspace MIMO-SAR system based on the burst mode imaging scheme utilizes multiple subpulses in a single PRI with the same carrier frequency and phase coding but with different time delays and transmitting beam pointing direction to obtain more spatial samples in azimuth. Therefore, the proposed imaging approach includes three important parts: DBF with null steering in elevation to separate echoes corresponding to different subpulses, azimuth multichannel reconstruction to resolve the azimuth nonuniform sampling, and a single-channel burst mode SAR imaging processor as shown in Figure 5.

3.1. Two-Step DBF with Null Steering in Elevation. According to above-mentioned analysis results, as shown in Figure 1, four subpulses are transmitted in a single PRI, and their corresponding echoes are simultaneously received and should be separated. Fortunately, echoes of subpulses transmitted to different subswaths can be easily separated via a narrow scanning pencil beam, since the angular interval between two illuminated subswaths as shown in Figure 1(b) is much larger than the beamwidth of the narrow scanning pencil beam. Therefore, this paper is just focused on separating echoes of subpulses transmitted to the same subswath.

As multiple subpulses are transmitted in a single PRI, their corresponding echoes will overlap at each receiver. For example, there are two point targets in the designed scene, point target $\mathrm{P}$ with the far slant range and point target $\mathrm{Q}$ with the near slant range as shown in Figure 2. Echoes of $\mathrm{P}$ corresponding to the first subpulse and $\mathrm{Q}$ corresponding to the second subpulse received by the first subaperture in elevation are, respectively, expressed as follows:

$$
\sigma_{1}(\tau)=\operatorname{rect}\left[\frac{\tau-\left(r_{t, 1}+r_{1}\right) / c}{\tau_{p}}\right]
$$




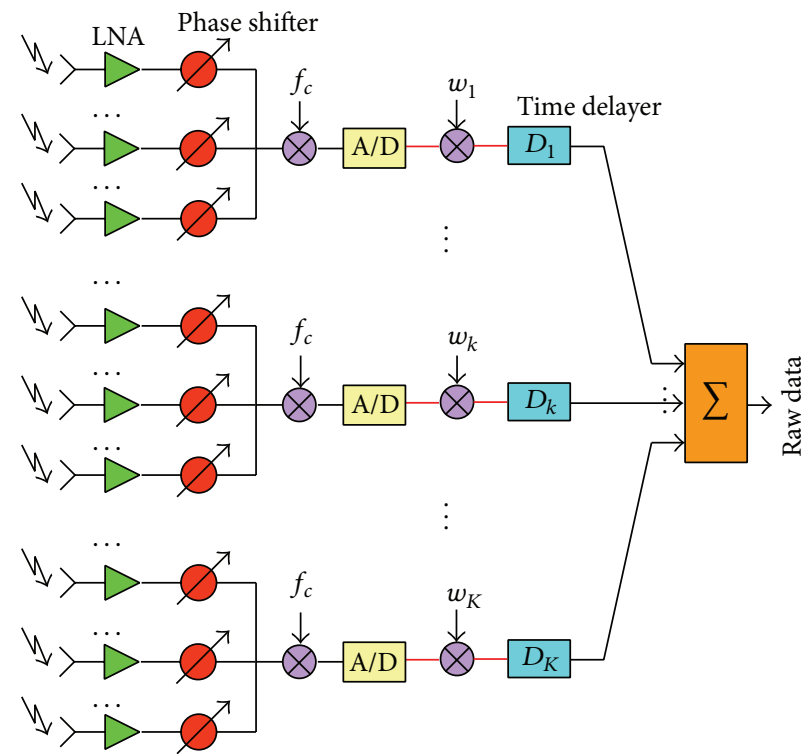

FIGURE 4: The block diagram of the real time conventional DBF processor in elevation onboard.

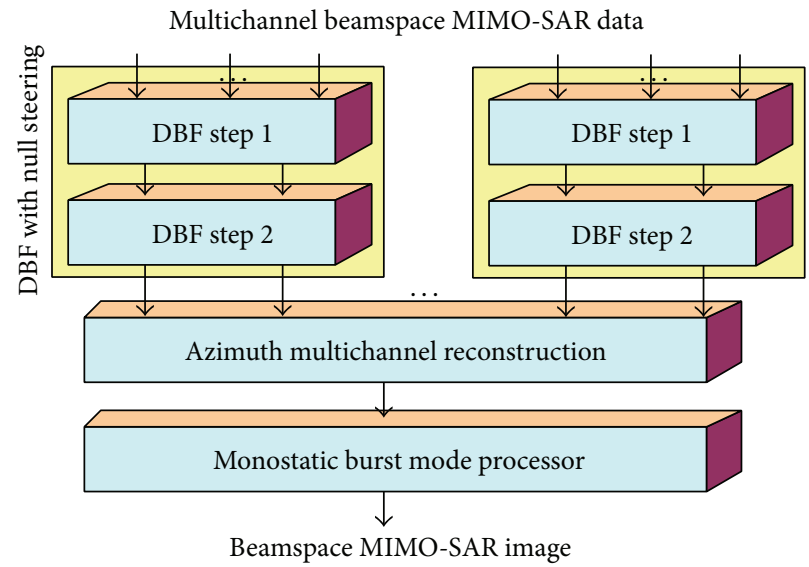

FIGURE 5: Block diagram of the proposed imaging approach.

$$
\begin{gathered}
\cdot \exp \left[j 2 \pi f_{c}\left(\tau-\frac{r_{t, 1}+r_{1}}{c}\right)\right. \\
\left.+j \pi k_{r}\left(\tau-\frac{r_{t, 1}+r_{1}}{c}\right)^{2}\right], \\
\sigma_{2}(\tau)=\operatorname{rect}\left[\frac{\tau-\tau_{d}-\left(r_{t, 2}+r_{2}\right) / c}{\tau_{p}}\right] \\
\cdot \exp \left[j 2 \pi f_{c}\left(\tau-\tau_{d}-\frac{r_{t, 2}+r_{2}}{c}\right)\right. \\
\left.+j \pi k_{r}\left(\tau-\tau_{d}-\frac{r_{t, 2}+r_{2}}{c}\right)^{2}\right],
\end{gathered}
$$

where $r_{t, 1}$ and $r_{t, 2}$ are the slant ranges from the transmitter to point targets $\mathrm{P}$ and $\mathrm{Q}$, respectively, while $r_{1}$ and $r_{2}$ are the slant ranges from the first receiving subaperture to point targets $\mathrm{P}$ and $Q$, respectively. The following equation demonstrates the slant range relationship between two point targets:

$$
\Delta r=\left(r_{t, 1}+r_{1}\right)-\left(r_{t, 2}+r_{2}\right)=\Delta \tau_{d} \cdot c .
$$

As a result, echoes of two subpulses from targets with different range locations completely overlap in the receiving window as shown in Figure 2. However, different ranges are associated with different looking angles in elevation. It is possible to separate simultaneously received echoes from different range bines by DBF on receive in elevation [9].

Echoes received by multiple subapertures and stored by individual channels in elevation could be expressed as a vector $\mathbf{s}(\tau)$ as follows:

$$
\mathbf{s}(\tau)=\left[s_{1}(\tau), s_{2}(\tau), \ldots, s_{K}(\tau)\right]^{T},
$$

where the symbol $(\cdot)^{T}$ denotes the transpose operator, $s_{k}(\tau),(k=1,2, \ldots, K)$ indicates the instantaneous received 
signal in the $k$ th subaperture, and $K$ is the number of subapertures in elevation. Assuming that two subpulses are transmitted in a subswath in a single PRI, the vector $\mathbf{s}(\tau)$ can be rewritten as follows:

$$
\begin{aligned}
\mathbf{s}(\tau) & =\sigma_{1}(\tau) \cdot \mathbf{u}_{1}(\tau)+\sigma_{2}(\tau) \cdot \mathbf{u}_{2}(\tau) \\
& =\left[\mathbf{u}_{1}(\tau), \mathbf{u}_{2}(\tau)\right]\left[\begin{array}{l}
\sigma_{1}(\tau) \\
\sigma_{2}(\tau)
\end{array}\right]
\end{aligned}
$$

with

$$
\begin{gathered}
\mathbf{u}_{1}(\tau)=\left[1, \exp \left(j \frac{2 \pi}{\lambda} d \cdot \sin \theta_{1}(\tau)\right), \ldots,\right. \\
\left.\exp \left(j \frac{2 \pi}{\lambda}(K-1) \cdot d \cdot \sin \theta_{1}(\tau)\right)\right]^{T}, \\
\mathbf{u}_{2}(\tau)=\left[1, \exp \left(j \frac{2 \pi}{\lambda} d \cdot \sin \theta_{2}(\tau)\right), \ldots,\right. \\
\left.\exp \left(j \frac{2 \pi}{\lambda}(K-1) \cdot d \cdot \sin \theta_{2}(\tau)\right)\right]^{T},
\end{gathered}
$$

where $\theta_{1}$ and $\theta_{2}$ are the off-boresight angles of point targets $\mathrm{P}$ and $\mathrm{Q}$, respectively. To separate echoes corresponding to different transmitted subpulses, the weighting matrix $\mathbf{w}(\tau)$ is multiplied to satisfy the following relationship:

$$
\begin{aligned}
{\left[\begin{array}{l}
\sigma_{1}(\tau) \\
\sigma_{2}(\tau)
\end{array}\right] } & =\mathbf{w}(\tau) \mathbf{s}(\tau) \\
& =\mathbf{w}(\tau)\left[\mathbf{u}_{1}(\tau), \mathbf{u}_{2}(\tau)\right]\left[\begin{array}{l}
\sigma_{1}(\tau) \\
\sigma_{2}(\tau)
\end{array}\right]
\end{aligned}
$$

with

$$
\begin{aligned}
\mathbf{w}(\tau) & =\left[\mathbf{w}_{1}(\tau), \mathbf{w}_{2}(\tau)\right]^{T}, \\
\mathbf{w}_{1}(\tau) & =\left[w_{1,1}(\tau), w_{1,2}(\tau), \ldots, w_{1, K}(\tau)\right], \\
\mathbf{w}_{2}(\tau) & =\left[w_{2,1}(\tau), w_{2,2}(\tau), \ldots, w_{2, K}(\tau)\right] .
\end{aligned}
$$

According to the matrix theory, the weighting matrix $\mathbf{w}(\tau)$ is obtained as follows:

$$
\mathbf{w}(\tau)=\mathbf{u}^{+}(\tau)=\left(\mathbf{u}^{H} \mathbf{u}\right)^{-1} \mathbf{u}^{H}
$$

with

$$
\mathbf{u}(\tau)=\left[\mathbf{u}_{1}(\tau), \mathbf{u}_{2}(\tau)\right]
$$

where $(\cdot)^{H}$ denotes the conjugate transpose operator, $(\cdot)^{-1}$ denotes the inverse operator, and $(\cdot)^{+}$denotes the pseudoinverse operator. Submitting (16) into (19) yields

$$
\begin{aligned}
\mathbf{w}(\tau) & =\mathbf{b}(\tau) \mathbf{a}(\tau)=\left[\begin{array}{ll}
b_{1,1}(\tau) & b_{1,2}(\tau) \\
b_{2,1}(\tau) & b_{2,2}(\tau)
\end{array}\right]\left[\begin{array}{l}
\mathbf{a}_{1}(\tau) \\
\mathbf{a}_{2}(\tau)
\end{array}\right] \\
& =\left[\begin{array}{ll}
c_{1,1}(\tau) & c_{1,2}(\tau) \\
c_{2,1}(\tau) & c_{2,2}(\tau)
\end{array}\right]^{-1}\left[\begin{array}{l}
\mathbf{a}_{1}(\tau) \\
\mathbf{a}_{2}(\tau)
\end{array}\right]
\end{aligned}
$$

with

$$
\begin{gathered}
\mathbf{a}_{1}(\tau)=\left[a_{1,1}, a_{1,2}, \ldots, a_{1, K}\right]=\left[1, \exp \left(-j \frac{2 \pi}{\lambda} d\right.\right. \\
\left.\cdot \sin \theta_{1}(\tau)\right), \ldots, \exp \left(-j \frac{2 \pi}{\lambda}(K-1) \cdot d\right. \\
\left.\left.\cdot \sin \theta_{1}(\tau)\right)\right]^{T},
\end{gathered}
$$

$$
\begin{aligned}
& \mathbf{a}_{2}(\tau)=\left[a_{2,1}, a_{2,2}, \ldots, a_{2, K}\right]=\left[1, \exp \left(-j \frac{2 \pi}{\lambda} d\right.\right. \\
& \left.\cdot \sin \theta_{2}\left(\tau_{k}\right)\right), \ldots, \exp \left(-j \frac{2 \pi}{\lambda}(K-1) \cdot d\right. \\
& \left.\left.\cdot \sin \theta_{2}\left(\tau_{k}\right)\right)\right]^{T}, \\
& c_{p, q}(\tau)=\sum_{k}^{K} \exp \left\{j \frac{2 \pi}{\lambda} d \cdot(k-1)\right. \\
& \left.\cdot\left[\sin \left(\theta_{q}(\tau)\right)-\sin \left(\theta_{p}(\tau)\right)\right]\right\}, \quad \text { with } p, q=1,2 .
\end{aligned}
$$

Therefore, the multiplied matrix $\mathbf{w}(\tau)$ for DBF with null steering includes two parts: the phase vector $\mathbf{a}(\tau)$ for conventional beam scanning the same as (5) and the second multiplied matrix $\mathbf{b}(\tau)$ for null steering. However, a set of time delayers are introduced after the multiplied phase vector for conventional beam scanning to compensate the spread of the signal over time of the chirp signal as shown in Figure 4. As a result, $c_{p, q}(\tau)$ is replaced by

$$
\begin{gathered}
c_{p, q}(\tau)=\sum_{k}^{K} \exp \left\{j \frac{2 \pi}{\lambda} d \cdot(k-1)\right. \\
\left.\cdot\left[\sin \left(\theta_{q}\left(\tau_{k}+D_{k}\right)\right)-\sin \left(\theta_{p}\left(\tau_{k}+D_{k}\right)\right)\right]\right\},
\end{gathered}
$$

with $p, q=1,2$.

The block diagram of the real time DBF processor with null steering in elevation onboard is shown in Figure 6. The processor includes three parts: the ABF step controlled by a series of phase shifters to receive echoes from different subswaths, the first DBF step to form two sharp beams to receive echoes from different subpulses implemented by the multiplied phase matrix $\mathbf{a}(\tau)$ and multiple time delayers, and the second DBF step to further separate echoes corresponding to different subpulses to extract different spatial diversities by null steering. Compared with the conventional real time $\mathrm{DBF}$ processor to separate echoes corresponding to different subpulses, the additional processing step of the proposed approach is the second multiplied matrix $\mathbf{b}(\tau)$ for null steering, and the second matrix $\mathbf{b}(\tau)$ multiplication is more easily handled due to the less resource consumption. 


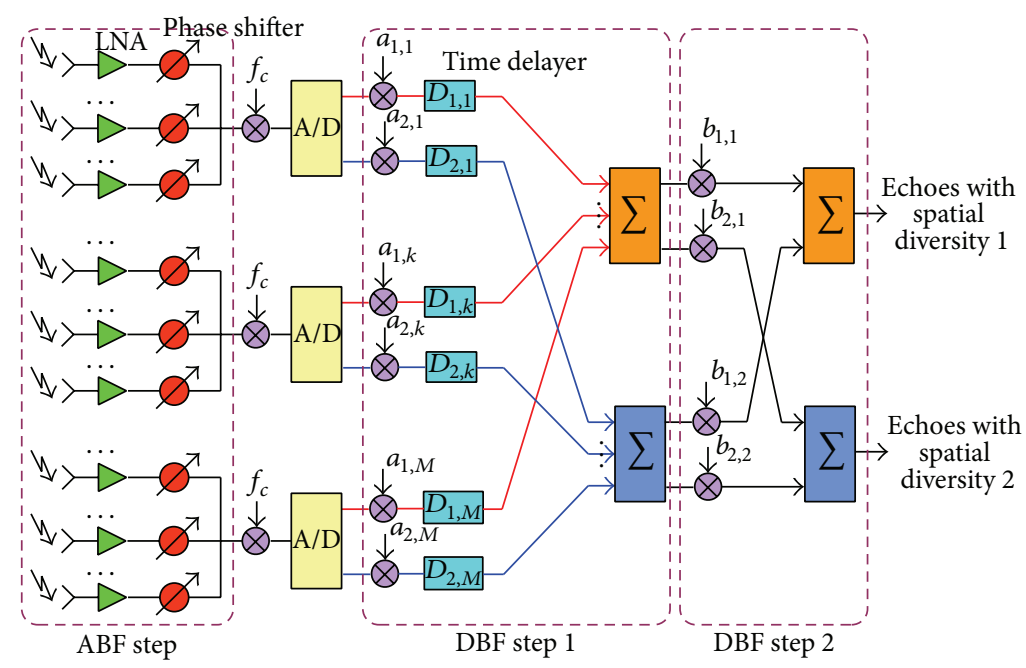

FIGURE 6: The block diagram of the real time DBF processor with null steering in elevation onboard.
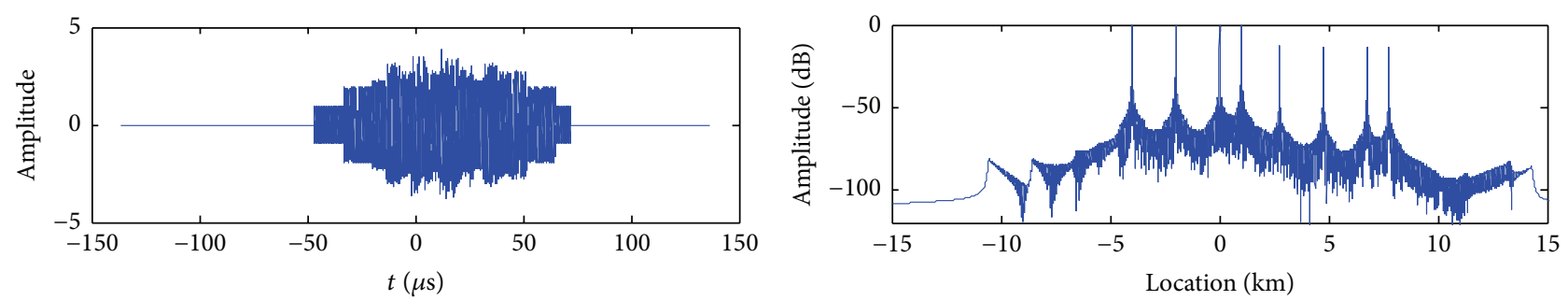

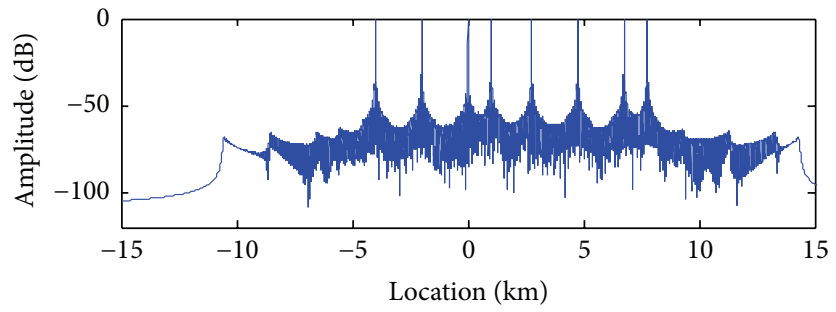

(a)

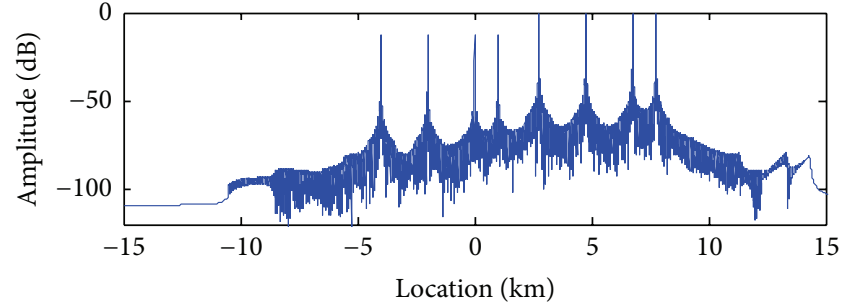

(b)
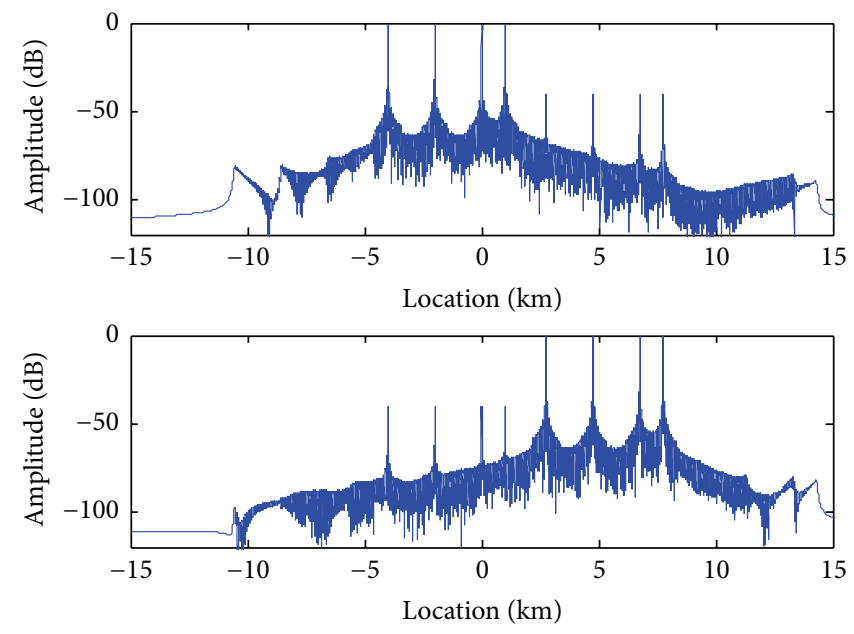

(c)

FiguRE 7: Echoes separation experiment. (a) Echo received by one of elevation receive channel (top) and range compressing results. (b) Echo separation results handled by the convectional DBF processor. (c) Echo separation results handled by the proposed two-step DBF processor. 


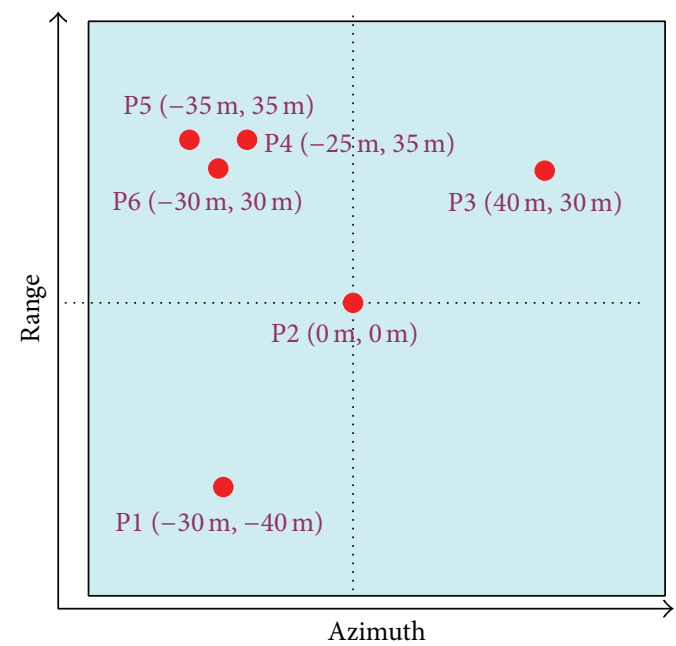

FIGURE 8: The designed imaged scene in the slant range plane with six point targets.

Furthermore, the fast time varying matrix $\mathbf{a}(\tau)$ could be computed on the ground and with uplink data transmission.

3.2. Azimuth Multichannel Reconstruction. After separating echoes corresponding to different subpulses in the same swath, echoes received by all azimuth subapertures should be combined together. Compared with the SIMO SAR system, more effective phase centers are obtained, since two transmitters are adopted as shown in Figure 1. The multichannel impulse response corresponding to the subpulse transmitted by Txl in azimuth as shown in Figure 1 can be written as follows:

$$
\begin{aligned}
h_{s, 1, n}(t) & =\exp \left[-j \frac{2 \pi}{\lambda}\left(\sqrt{v^{2} t^{2}+r^{2}}+\sqrt{\left(v t-\Delta x_{n}\right)^{2}+r^{2}}\right)\right] \\
= & \exp \left[-j \frac{4 \pi}{\lambda} r\right] \cdot \exp \left[-j \frac{\pi \cdot \Delta x_{n}^{2}}{2 \lambda r}\right] \\
& \cdot \exp \left[-j \frac{2 \pi v^{2}}{\lambda r}\left(t-\frac{\Delta x_{n}}{2 v}\right)^{2}\right] \\
= & h_{s}\left(t-\frac{\Delta x_{n}}{2 v}\right) \cdot \exp \left[-j \frac{\pi \cdot \Delta x_{n}^{2}}{2 \lambda r}\right]
\end{aligned}
$$

with

$$
h_{s}(t)=\exp \left[-j \frac{4 \pi}{\lambda} r\right] \cdot \exp \left[-j \frac{2 \pi v^{2}}{\lambda r} t^{2}\right]
$$

where $r$ is the effective slant range, $v$ is the effective velocity of SAR sensor, $\Delta x_{n}$ is the physical spacing between Txl and the $n$th azimuth subaperture along the track, and $h_{s}(t)$ indicates the impulse response of the single-channel case. Furthermore, the multichannel impulse response corresponding to the subpulse transmitted by $\mathrm{Tx} 2$ in azimuth as shown in Figure 1 can be written as

$$
\begin{aligned}
& h_{s, 2, n}(t)=\exp [-j \\
& \left.\cdot \frac{2 \pi}{\lambda}\left(\sqrt{\left(v t-\Delta x_{N}\right)^{2}+r^{2}}+\sqrt{\left(v t-\Delta x_{n}\right)^{2}+r^{2}}\right)\right] \\
& \approx \exp \left[-j \frac{4 \pi}{\lambda} r\right] \cdot \exp \left[-j \frac{\pi \cdot\left(\Delta x_{N}-\Delta x_{n}\right)^{2}}{2 \lambda r}\right] \\
& \cdot \exp \left[-j \frac{2 \pi v^{2}}{\lambda r}\left(t-\frac{\Delta x_{n}+\Delta x_{N}}{2 v}\right)^{2}\right]=h_{s}(t \\
& \left.-\frac{\Delta x_{n}+\Delta x_{N}}{2 v}\right) \cdot \exp \left[-j \frac{\pi \cdot\left(\Delta x_{N}-\Delta x_{n}\right)^{2}}{2 \lambda r}\right] .
\end{aligned}
$$

Furthermore, $\Delta x_{n}=(n-1) \cdot \Delta x$ is assumed as shown in Figure 1 in this paper; the multichannel impulse responses $h_{s, 1, n}(t)$ and $h_{s, 2, n}(t)$ could be rewritten as follows:

$$
\begin{aligned}
h_{s, 1, n}(t)= & h_{s}\left(t-\frac{(n-1) \cdot \Delta x}{2 v}\right) \\
\cdot & \exp \left[-j \frac{\pi \cdot(n-1)^{2} \cdot \Delta x^{2}}{2 \lambda r}\right], \\
h_{s, 2, n}(t)= & h_{s}\left(t-\frac{(n+N-2) \cdot \Delta x}{2 v}\right) \\
& \cdot \exp \left[-j \frac{\pi \cdot(N-n)^{2} \cdot \Delta x^{2}}{2 \lambda r}\right] .
\end{aligned}
$$

From the comparison between (27) and (28), the following relation is obtained as follows:

$$
h_{s, 2,1}(t)=h_{s, 1, N}(t) .
$$

Consequently, the number of effective phase centers in the presented beamspace MIMO-SAR system with two transmit 


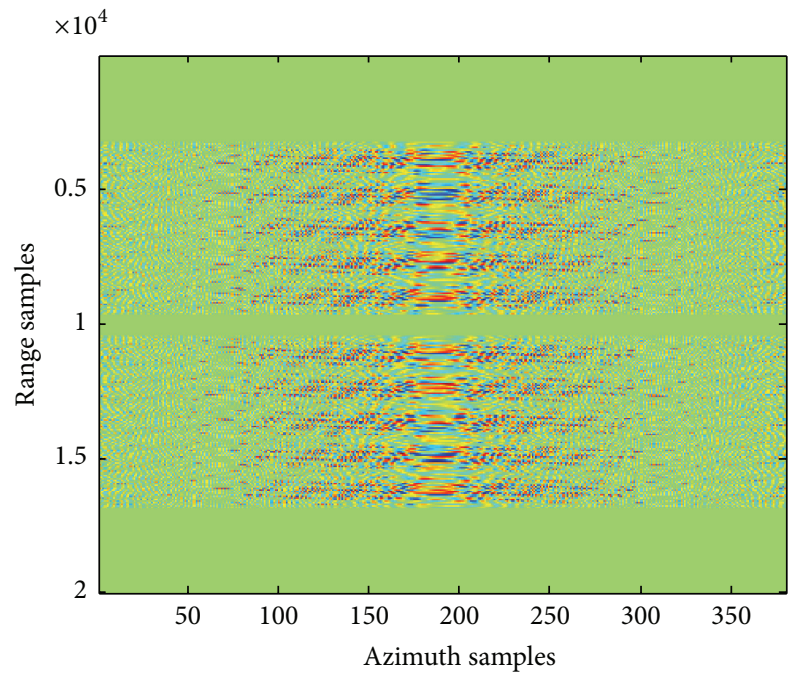

(a)

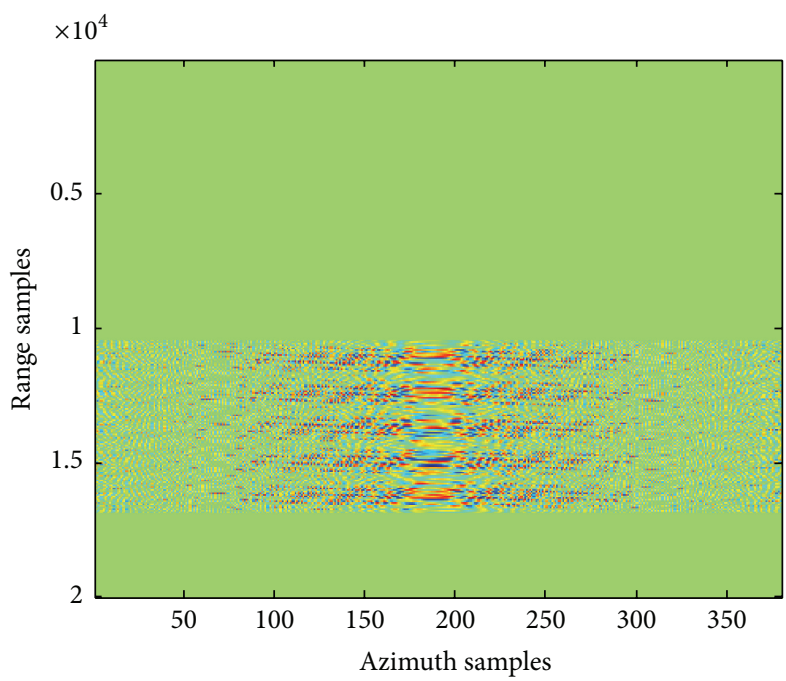

(c)

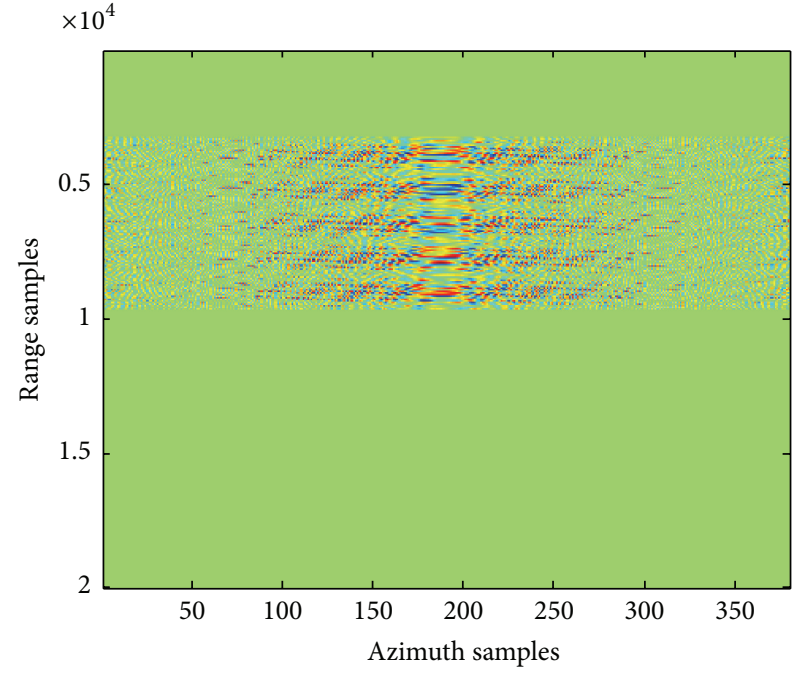

(b)

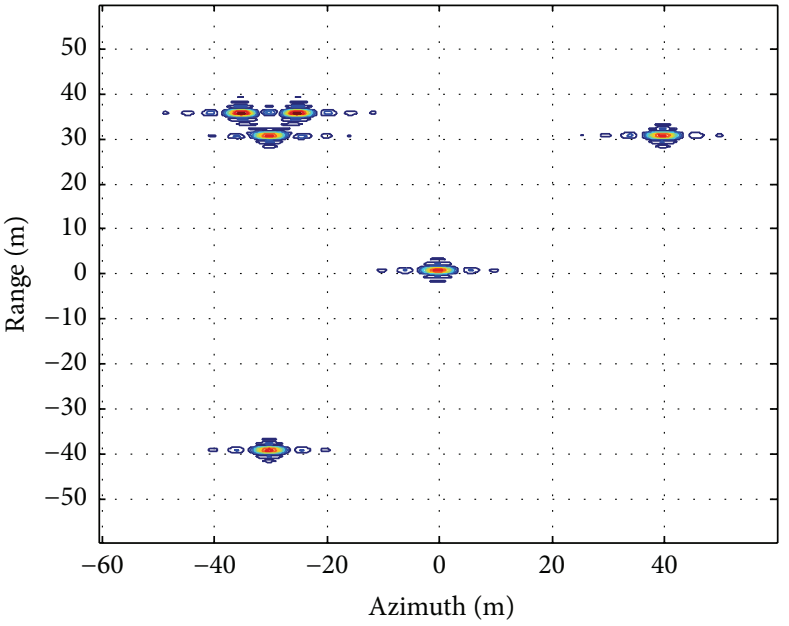

(d)

FIGURE 9: Simulation results of the designed scene. (a) Echoes received by one of the subapertures. (b) Echoes of the imaged scene corresponding to the first subpulse after DBF with null steering. (c) Echoes of the imaged scene corresponding to the second subpulse after DBF with null steering. (d) Two-dimension spectrum of the imaged scene.

subapertures and $N$ receive subapertures is $2 N-1$, and the optimum PRF for azimuth uniform sampling is

$$
\mathrm{PRF}_{\mathrm{opt}}=\frac{2 v}{(2 N-1) \cdot \Delta x}
$$

As a result, such azimuth multichannel SAR system is characterized by the matrix $\mathbf{H}\left(f_{a}\right)$ as follows:

$$
\mathbf{H}\left(f_{a}\right)=\left[\begin{array}{ccc}
H_{1}\left(f_{a}\right) & \cdots & H_{2 N}\left(f_{a}\right) \\
\vdots & \ddots & \vdots \\
H_{1}\left(f_{a}+(2 N-2) \cdot \mathrm{PRF}\right) & \cdots & H_{2 N}\left(f_{a}+(2 N-2) \cdot \mathrm{PRF}\right)
\end{array}\right]_{(2 N-1) \times 2 N}
$$

with 


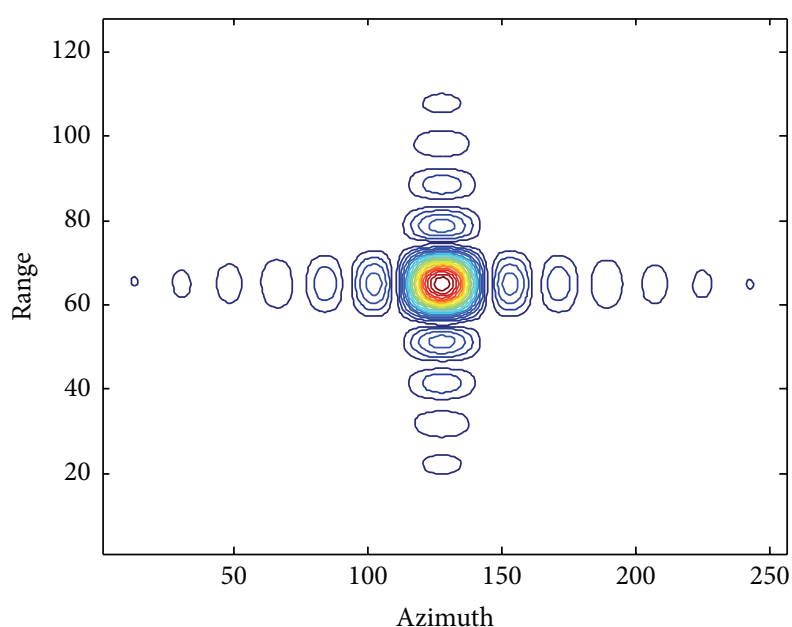

(a)

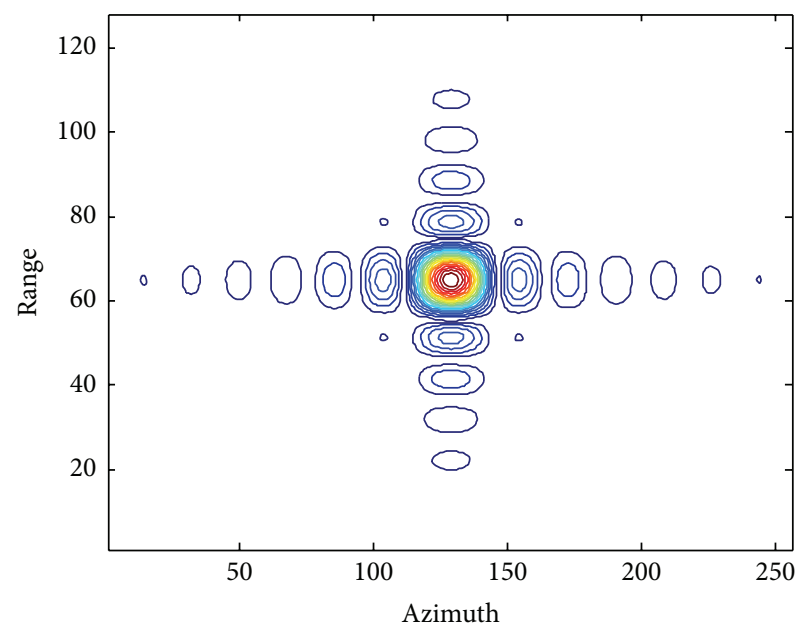

(b)

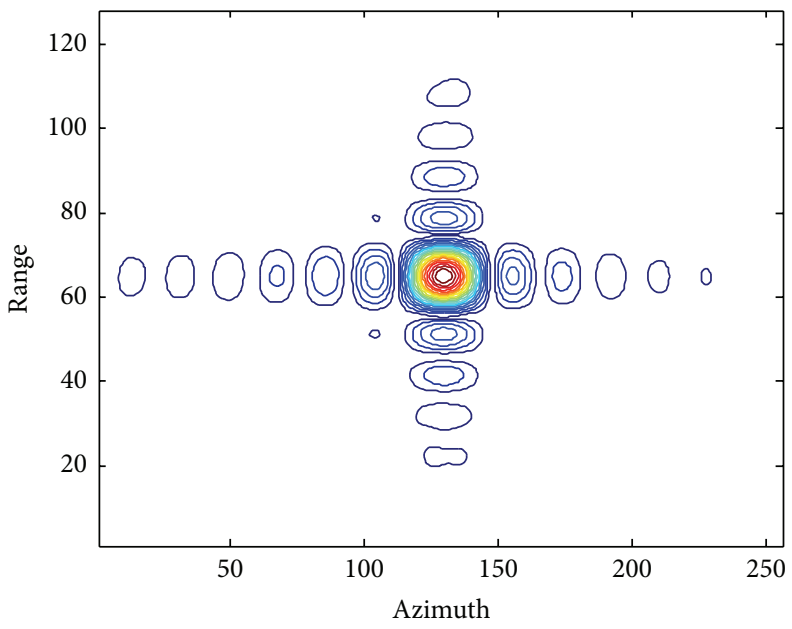

(c)

Figure 10: Contour plots of three point targets. (a) P1. (b) P2. (c) P3.

$$
H_{n}\left(f_{a}\right)= \begin{cases}\exp \left[-j \frac{\pi \cdot(n-1)^{2} \cdot \Delta x^{2}}{2 \lambda r}\right] \cdot \exp \left[-j \cdot 2 \pi \cdot f_{a} \cdot \frac{(n-1) \cdot \Delta x}{2 v}\right], & \text { with } n \leq N, \\ \exp \left[-j \frac{\pi \cdot(2 N-n-1)^{2} \cdot \Delta x^{2}}{2 \lambda r}\right] \cdot \exp \left[-j \cdot 2 \pi \cdot f_{a} \cdot \frac{(n-1) \cdot \Delta x}{2 v}\right] . & \text { with } n>N .\end{cases}
$$

It should be noted that the multichannel signal with the bandwidth of $(2 N-1)$. PRF only could be recovered in such system, since two azimuth channels are with the same azimuth spatial phase center. Consequently, the multichannel signal reconstruction matrix could be easily obtained as follows:

$$
\mathbf{P}\left(f_{a}\right)=\mathbf{H}^{+}\left(f_{a}\right)=\left(\mathbf{H}^{H}\left(f_{a}\right) \mathbf{H}\left(f_{a}\right)\right)^{-1} \mathbf{H}^{H}\left(f_{a}\right) .
$$

With the multichannel reconstruction matrix $\mathbf{P}\left(f_{a}\right)$, the reconstructed azimuth multichannel raw data could be considered as the single-channel signal with the sampling rate of $(2 N-1) \cdot$ PRF.
As the mentioned beamspace MIMO-SAR is mainly based on the burst imaging mode. The resulting raw data after DBF in elevation and azimuth multichannel reconstruction could be handled by burst mode (ScanSAR or TOPS) SAR processors.

\section{Simulation Experiment}

To validate the proposed imaging approach, experiments on simulated raw data are carried out. Simulation parameters are listed in Table 1. 


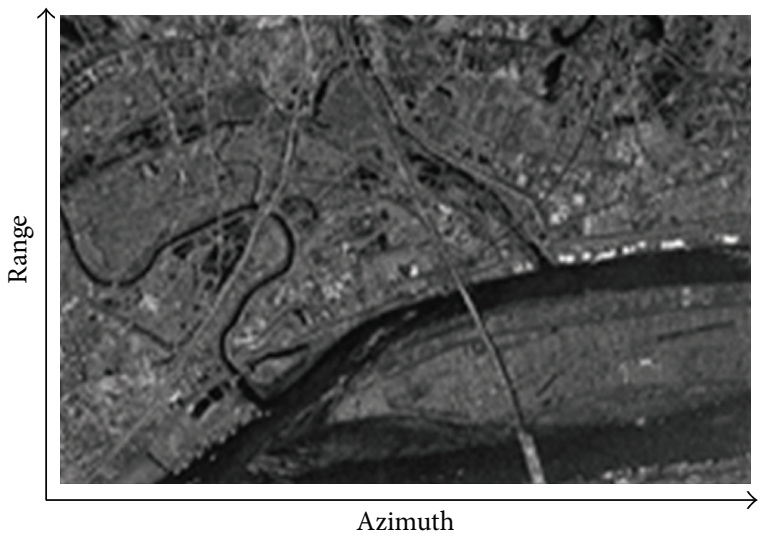

(a)

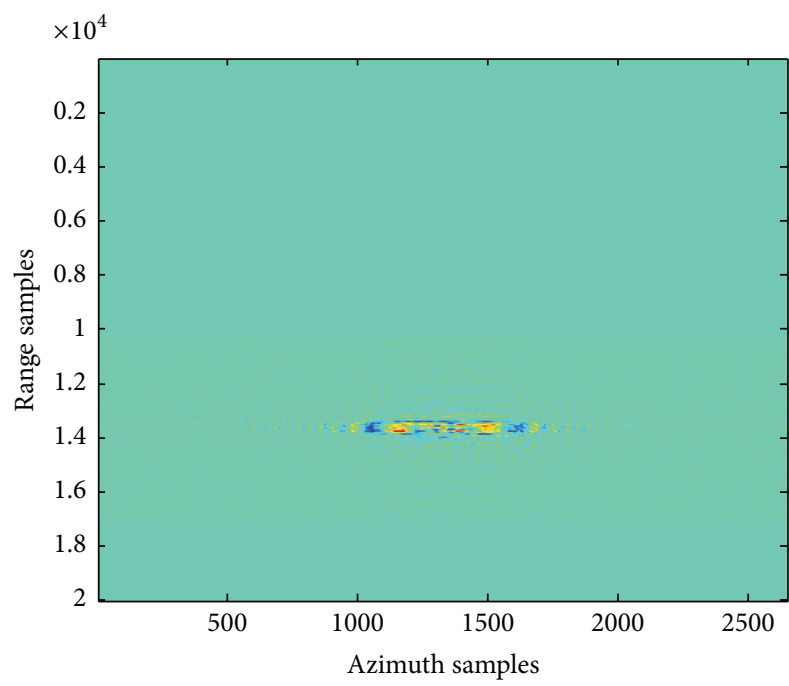

(c)

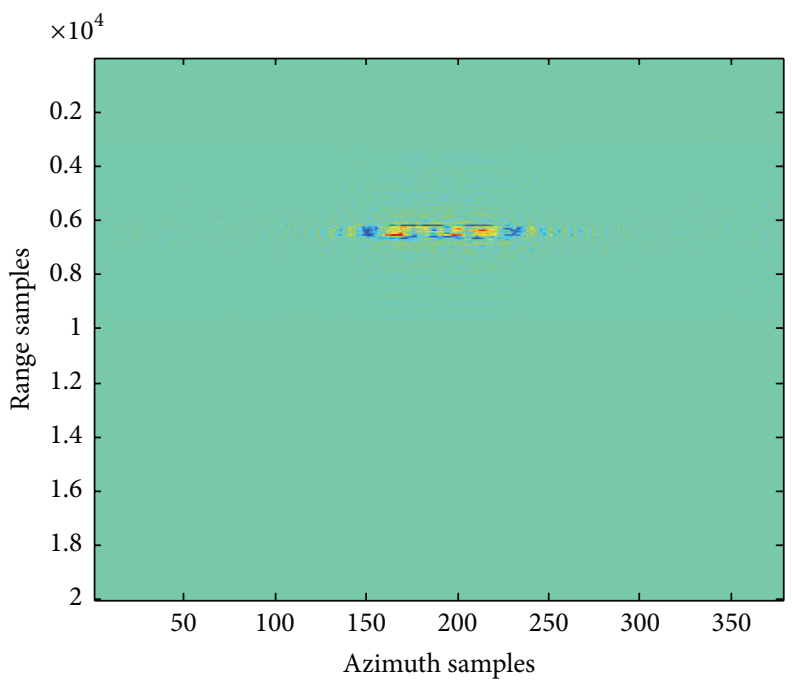

(b)

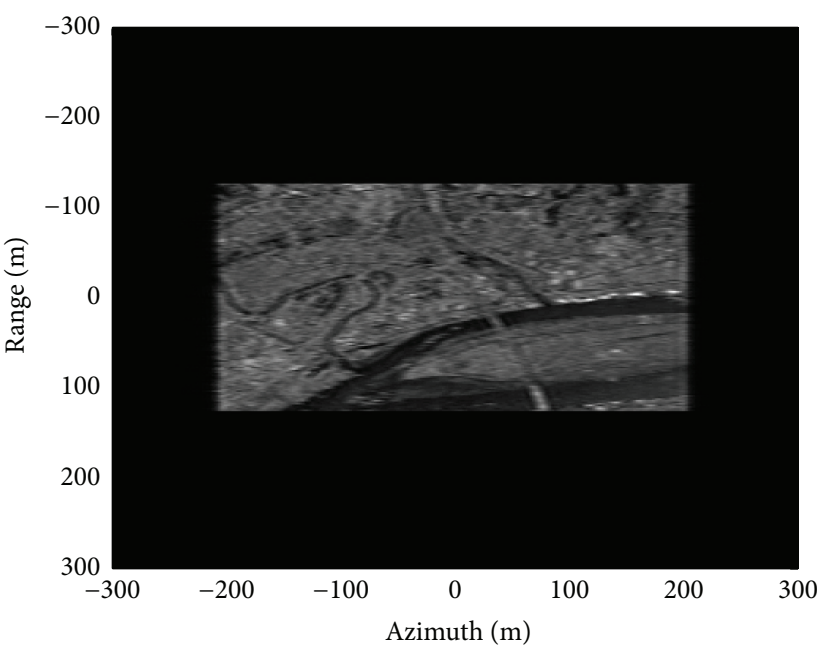

(d)

FigurE 11: Simulation results of the designed distributed scene. (a) The designed extended scene. (b) Echoes of the imaged scene corresponding to the first subpulse after DBF with null steering. (c) Echoes of the imaged scene corresponding to the second subpulse after DBF with null steering. (d) Two-dimension spectrum of the imaged scene.

Figure 7 compares the echoes separating capacities of the conventional DBF processor and the proposed two-step DBF processor. As shown in Figures 7(b) and 7(c), simulation results demonstrate the advantage of the proposed approach about $20 \mathrm{~dB}$ better than the conventional DBF processor. In this simulation, four point targets with different range locations are designed.

To validate the whole processor imaging processor, an imaging scene consisting of six point targets is designed as shown in Figure 8. Figure 9(a) shows echoes of the designed scene received by one of the elevation receive channels, and echoes corresponding to different subpulses are received by each receive channel in both azimuth and elevation. After DBF processing with null steering, echoes corresponding to different subpulses are well separated as shown in Figures 9(b) and 9(c). The simulation results validate the separating effect of the modified DBF processor with null steering. Without using any window functions, the final imaging result of the designed scene is shown in Figure 9(d). Furthermore, interpolated contour plots of three of the processed point targets with different range bines show the well focused features and the well-behavior sidelobes, and their imaging parameters are evaluated and listed in Table 2. Both Figure 10 and Table 2 validate that the DBF processor and the modified azimuth multichannel reconstruction algorithm would not affect the following burst mode SAR imaging. To further validate the proposed imaging approach, a distributed imaging scene is designed as shown in Figure 11(a). The space between adjacent pixels in both azimuth and range directions is $0.7 \mathrm{~m}$, which is smaller than both azimuth and range resolutions. 
TABLE 1: Simulation parameter.

\begin{tabular}{lr}
\hline Parameter & Value \\
\hline Satellite height & $570 \mathrm{~km}$ \\
Satellite velocity & $7585 \mathrm{~m} / \mathrm{s}$ \\
Subpulse duration & $35 \mu \mathrm{s}$ \\
Bandwidth & $150 \mathrm{MHz}$ \\
Sampling frequency & $180 \mathrm{MHz}$ \\
Time delay between two subpulses & $40 \mu \mathrm{s}$ \\
Operated PRF & $1180 \mathrm{~Hz}$ \\
Number of azimuth subapertures & 4 \\
Number of elevation subapertures & 10 \\
Subaperture height & $0.2 \mathrm{~m}$ \\
Subaperture length & $2.4 \mathrm{~m}$ \\
Look angle & $28.6^{\circ}$ \\
Slant range of the scene center & $634.68 \mathrm{~km}$ \\
Burst duration & $0.32 \mathrm{~s}$ \\
\hline
\end{tabular}

TABLE 2: Imaging parameters of simulated point targets.

\begin{tabular}{|c|c|c|c|c|c|c|}
\hline \multirow{2}{*}{ Targets } & \multicolumn{3}{|c|}{ Azimuth } & \multicolumn{3}{|c|}{ Range } \\
\hline & Resolution (m) & PSLR (dB) & ISLR (dB) & Resolution (m) & PSLR (dB) & $\operatorname{ISLR}(\mathrm{dB})$ \\
\hline P1 & 3.26 & -13.26 & -10.08 & 0.89 & -13.26 & -9.92 \\
\hline $\mathrm{P} 2$ & 3.27 & -13.26 & -10.02 & 0.89 & -13.26 & -9.98 \\
\hline P3 & 3.27 & -13.24 & -9.96 & 0.89 & -13.26 & -9.93 \\
\hline
\end{tabular}

\section{Conclusion}

In this paper, an imaging processor to handle the beamspace MIMO-SAR raw data is proposed. The key point of the proposed imaging processor is its echoes separation corresponding to different subpulses and azimuth multichannel raw data construction. Based on the relationship between the transmitted time delay and the SAR side-looking imaging geometry, echoes corresponding to different subpulses are separated by a DBF processor with null steering onboard. In the proposed DBF processor, echoes corresponding to different subpulses are received by different sharp scanning pencil beams, which are implemented by the steering matrix and a series of time delayers. Afterwards, the interference signal corresponding to the undesired subpulse received by the sidelobe of the sharp scanning pencil beam is further suppressed via the matrix of the second DBF step for null steering. Then, the azimuth multichannel raw data is reconstructed by a modified multichannel reconstruction filter according to the subaperture arrangement in azimuth of beamspace MIMO-SAR. Imaging results on simulated raw data validate the proposed imaging approach.

\section{Conflict of Interests}

The authors declare that there is no conflict of interests regarding the publication of this paper.

\section{Acknowledgment}

This work is supported by NSF of China (no. 61271177).

\section{References}

[1] N. Gebert, Multi-channel azimuth processing for high-resolution wide-swath SAR imaging [Ph.D. Dissertation], Universität Karlsruhe, Karlsruhe, Germany, 2009.

[2] A. Freeman, W. T. K. Johnson, B. Huneycutt et al., "The 'myth' of the minimum SAR antenna area constraint," IEEE Transactions on Geoscience and Remote Sensing, vol. 38, no. 1, pp. 320-324, 2000.

[3] B. R. Jean and J. W. Rouse Jr., "A multiple beam synthetic aperture radar design concept for geoscience applications," IEEE Transactions on Geoscience and Remote Sensing, vol. 21, no. 2, pp. 201-207, 1983.

[4] A. Currie and M. A. Brown, "Wide-swath SAR," IEE Proceedings, Part F: Radar and Signal Processing, vol. 139, no. 2, pp. 122135, 1992.

[5] G. D. Callaghan and I. D. Longstaff, "Wide-swath space-borne SAR using a quad-element array," IEE Proceedings: Radar, Sonar and Navigation, vol. 146, no. 3, pp. 159-165, 1999.

[6] M. Suess, B. Grafmueller, and R. Zahn, "A novel high resolution, wide swath SAR system," in Proceedings of the International Geoscience and Remote Sensing Symposium (IGARRS '01), pp. 1013-1015, Sydney, Australia, July 2001.

[7] M. Suess and W. Wiesbeck, "Side-looking synthetic aperture radar system," Euro Patent EP 1241487 A1, 2001.

[8] G. Krieger, N. Gebert, M. Younis, and A. Moreira, "Advanced synthetic aperture radar based on digital beamforming and waveform diversity," in Proceedings of the IEEE Radar Conference, pp. 1-6, Rome, Italy, May 2008.

[9] G. Krieger, N. Gebert, and A. Moreira, "Multidimensional waveform encoding: a new digital beamforming technique for 
synthetic aperture radar remote sensing," IEEE Transactions on Geoscience and Remote Sensing, vol. 46, no. 1, pp. 31-46, 2008.

[10] W.-Q. Wang, "Space-time coding MIMO-OFDM SAR for highresolution imaging," IEEE Transactions on Geoscience and Remote Sensing, vol. 49, no. 8, pp. 3094-3104, 2011.

[11] W.-Q. Wang, "Virtual antenna array analysis for MIMO synthetic aperture radars," International Journal of Antennas and Propagation, vol. 2012, Article ID 587276, 10 pages, 2012.

[12] W.-Q. Wang, "Mitigating range ambiguities in high-PRF SAR with OFDM waveform diversity," IEEE Geoscience and Remote Sensing Letters, vol. 10, no. 1, pp. 101-105, 2013.

[13] D. Cristallini, D. Pastina, and P. Lombardo, "Exploiting MIMO SAR potentialities with efficient cross-track constellation configurations for improved range resolution," IEEE Transactions on Geoscience and Remote Sensing, vol. 49, no. 1, pp. 38-52, 2011.

[14] G. Krieger, "MIMO-SAR: opportunities and pitfalls," IEEE Transactions on Geoscience and Remote Sensing, vol. 52, no. 5, pp. 2628-2645, 2014.

[15] W. Xu, Y. Deng, and R. Wang, "Multichannel synthetic aperture radar systems with a planar antenna for future spaceborne microwave remote sensing," IEEE Aerospace and Electronic Systems Magazine, vol. 27, no. 12, pp. 26-30, 2012.

[16] D. Garmatyuk, "Cross-range SAR reconstruction with multicarrier OFDM signals," IEEE Geoscience and Remote Sensing Letters, vol. 9, no. 5, pp. 808-812, 2012.

[17] J.-H. Kim, M. Younis, A. Moreira, and W. Wiesbeck, "A novel OFDM chirp waveform scheme for use of multiple transmitters in SAR," IEEE Geoscience and Remote Sensing Letters, vol. 10, no. 3, pp. 568-572, 2013.

[18] G. Krieger, N. Gebert, and A. Moreira, "Unambiguous SAR signal re-construction from non-uniform displaced phase center sampling," IEEE Geoscience and Remote Sensing Letters, vol. 1, no. 4, pp. 260-264, 2004.

[19] N. Gebert, G. Krieger, and A. Moreira, "Digital beamforming on receive: techniques and optimization strategies for highresolution wide-swath SAR imaging," IEEE Transactions on Aerospace and Electronic Systems, vol. 45, no. 2, pp. 564-592, 2009.

[20] A. Moreira, J. Mittermayer, and R. Scheiber, "Extended chirp scaling algorithm for air- and spaceborne SAR data processing in stripmap and ScanSAR imaging modes," IEEE Transactions on Geoscience and Remote Sensing, vol. 34, no. 5, pp. 1123-1136, 1996.

[21] R. Lanari, S. Hensley, and P. A. Rosen, "Chirp z-transform based SPECAN approach for phase-preserving ScanSAR image generation," IEE Proceedings-Radar, Sonar and Navigation, vol. 145 , no. 5, pp. 254-261, 1998.

[22] F. De Zan and A. M. Monti Guarnieri, "TOPSAR: terrain observation by progressive scans," IEEE Transactions on Geoscience and Remote Sensing, vol. 44, no. 9, pp. 2352-2360, 2006.

[23] P. Prats, R. Scheiber, J. Mittermayer, A. Meta, and A. Moreira, "Processing of sliding spotlight and TOPS SAR data using baseband azimuth scaling," IEEE Transactions on Geoscience and Remote Sensing, vol. 48, no. 2, pp. 770-780, 2010.

[24] W. Xu, P. Huang, R. Wang, Y. Deng, and Y. Lu, "TOPS-mode raw data processing using chirp scaling algorithm," IEEE Journal of Selected Topics in Applied Earth Observations and Remote Sensing, vol. 7, no. 1, pp. 235-246, 2014.

[25] W. Xu, P. Huang, R. Wang, and Y. Deng, "Processing of multichannel sliding spotlight and TOPS synthetic aperture radar data," IEEE Transactions on Aerospace and Electronic Systems, vol. 49, no. 3, pp. 2035-2045, 2013.
[26] F. Feng, S. Li, W. Yu, P. Huang, and W. Xu, "Echo separation in multi-dimensional waveform encoding SAR remote sensing using an advanced null-steering beamformer," IEEE Transactions on Geoscience and Remote Sensing, vol. 50, no. 10, pp. 41574172, 2012. 


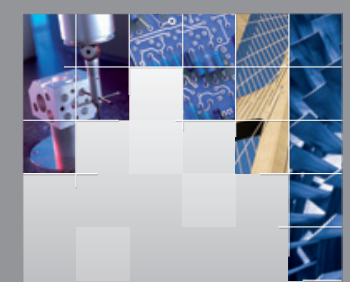

\section{Enfincering}
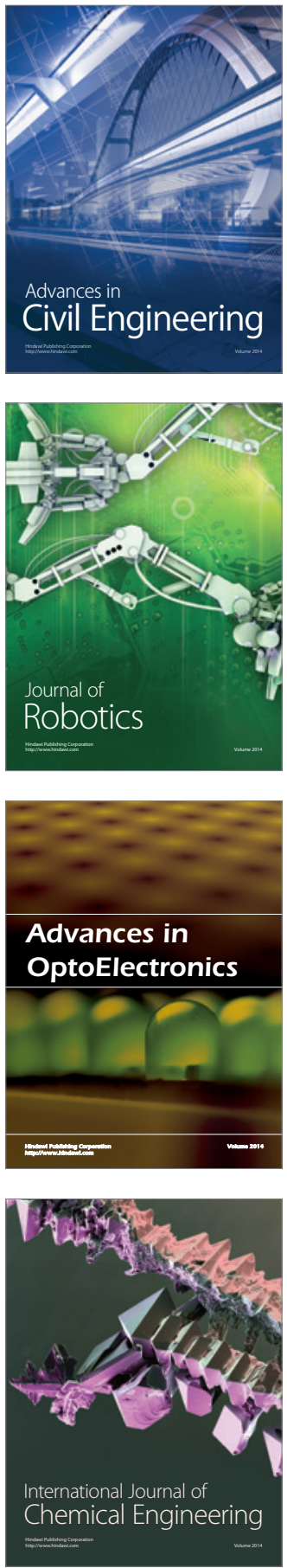

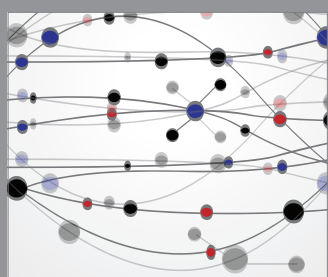

The Scientific World Journal

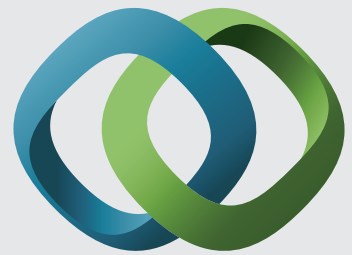

\section{Hindawi}

Submit your manuscripts at

http://www.hindawi.com
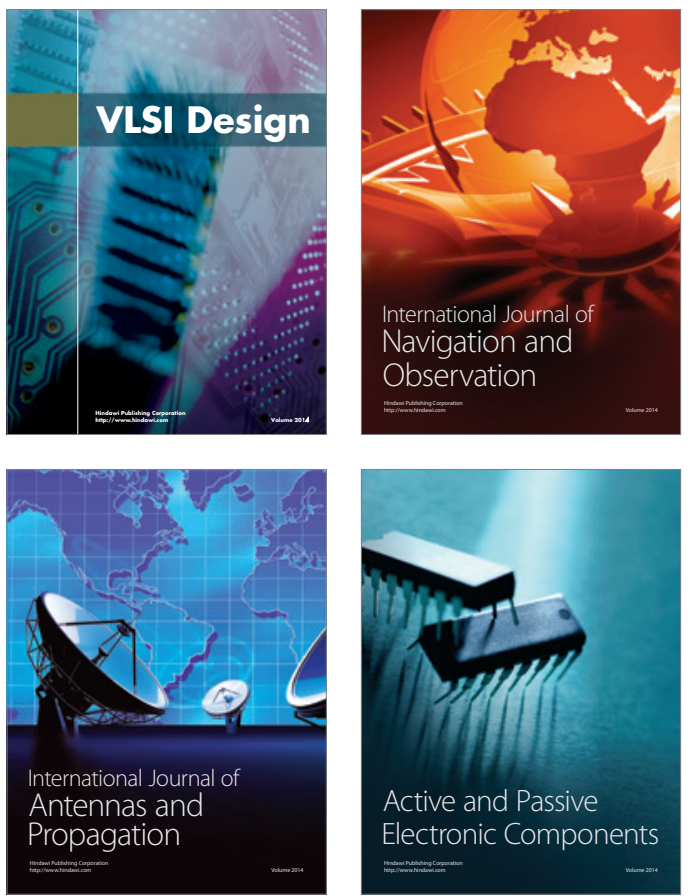
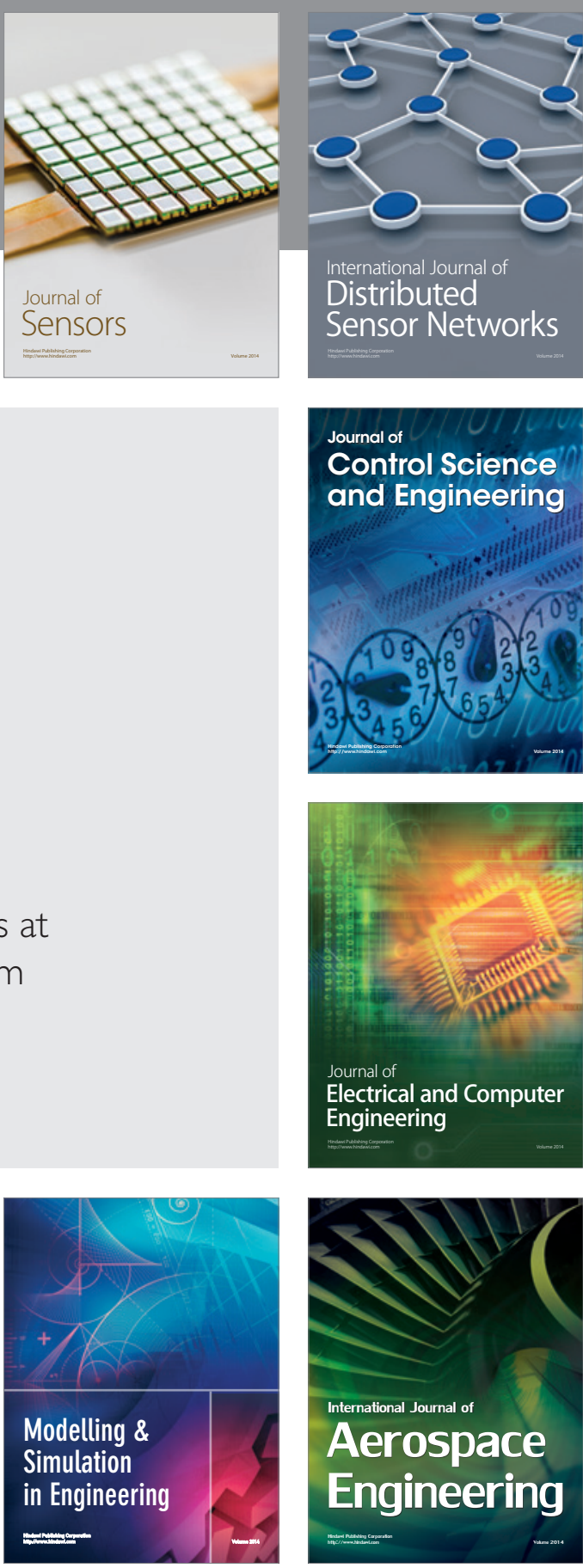

International Journal of

Distributed

Sensor Networks

Journal of

Control Science

and Engineering
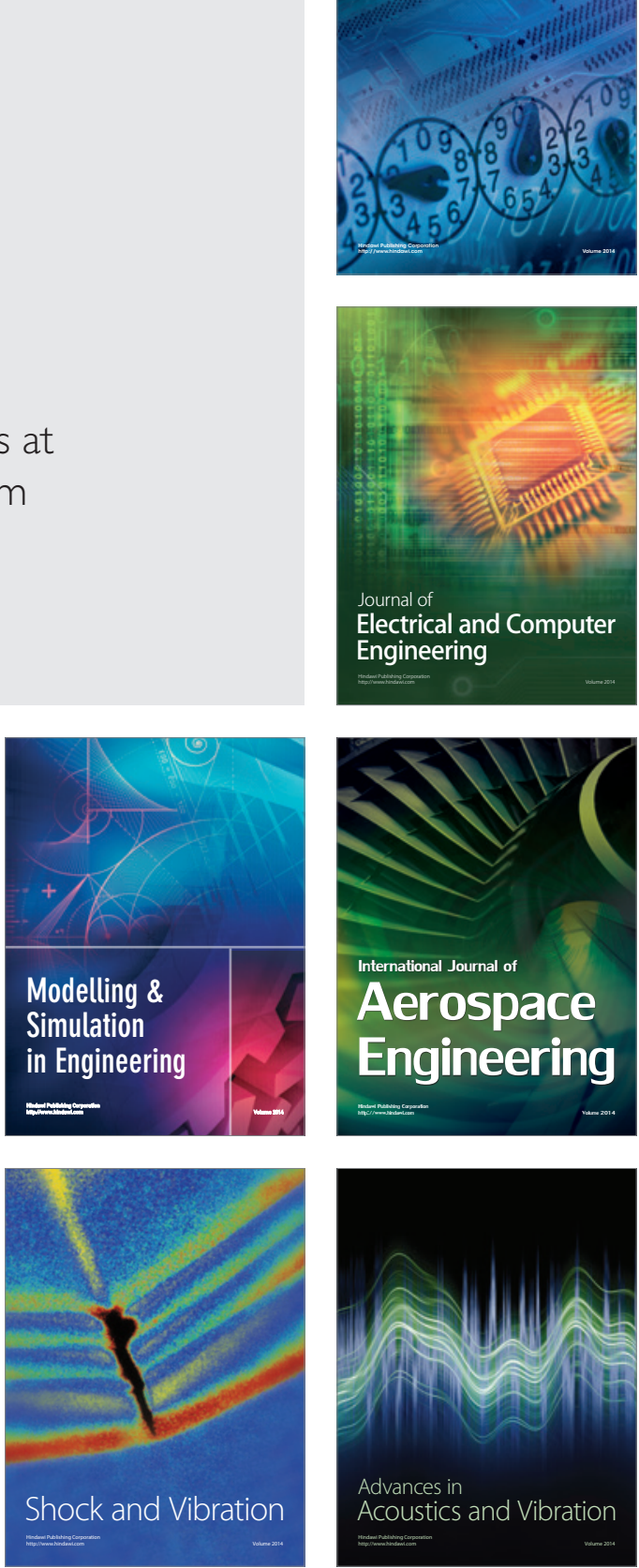